\title{
Sarcoglycans and integrins in bisphosphonate treatment: Immunohistochemical and scanning electron microscopy study
}

\author{
FRANCESCO SAVERIO DE PONTE ${ }^{1}$, ANGELO FAVALORO ${ }^{2,3}$, ENRICO NASTRO SINISCALCHI ${ }^{1}$, \\ ANTONIO CENTOFANTI ${ }^{2,3}$, MICHELE RUNCI ${ }^{1}$, GIUSEPPINA CUTRONEO ${ }^{2,3}$ and LUCIANO CATALFAMO ${ }^{1}$ \\ Departments of ${ }^{1}$ Experimental Medical, Surgical and Odontostomatological Sciences, \\ and ${ }^{2}$ Biomedical Sciences and Morpho-functional Imaging, University of Messina, Messina; \\ ${ }^{3}$ IRCCS, Centro Neurolesi 'Bonino Pulejo', Messina, Italy
}

Received April 1, 2013; Accepted May 17, 2013

DOI: $10.3892 /$ or.2013.2766

\begin{abstract}
Osteonecrosis of the jaw is an adverse outcome associated with bisphosphonate treatment. Bisphosphonates are used in conjunction with antineoplastic chemotherapy for the treatment of hypercalcaemia associated with malignancy, lytic bone metastasis and multiple myeloma. However, it is not known if the osteonecrosis of the jaw lesion originates in the bone or whether it initiates in the gingival epithelium. Two bisphosphonates are commonly used in cancer treatment. One of these is pamidronate disodium, a second-generation bisphosphonate that differs from the first-generation drug because it inhibits bone resorption at a dose that does not affect bone mineralization. The other widely used BP, zoledronate, is a third-generation drug that is the most potent bisphosphonate in clinical use, showing strong anti-osteoclastic activity, similar to pamidronate. The aim of the present study was to evaluate the modifications of human oral mucosa and underlying bone in patients after treatment with these nitrogen-containing bisphosphonates for 24 and 36 months. We analyzed the structural damage of the oral mucosa and damage of the perilesional mandibular bone observing possible correlations from them. Our results allow to express two hypotheses about the mechanism responsible for these results relating to mandible matrix necrosis; first, an increased skeletal microdamage associated with turnover suppression occurred early in treatment and progress with longer treatment duration, second, opening damage in osteonecrosis of the jaw modifies structural morphology of gingival epithelium.
\end{abstract}

Correspondence to: Professor Giuseppina Cutroneo, Department of Biomedical Sciences and of Morpho-functional Imaging, University of Messina, Via Consolare Valeria 1, I-98125 Messina, Italy

E-mail: gcutroneo@unime.it; anapuc@unime.it

Key words: gingival mucosa, bisphosphonates, bone tissue, osteonecrosis, sarcoglycans, integrins, scanning electron microscopy, immunohistochemistry

\section{Introduction}

Bisphosphonates (BPs) are stable analogues of pyrophosphate with P-C-P structure and 2 side chains attached to the carbon atom. BPs, approved for clinical use, differ based on structural alterations of the so called R-2 side chain, which determines the efficiency as inhibitor of bone resorption $(1,2)$. Drug potency has increased with each successive generation, as the R-2 side chain was lengthened and an amino group incorporated. Until now, osteonecrosis of the jaws (ONJ) has been described only in patients undergoing treatment with amino-group-containing BPs, which are much more potent than of that the non-amino group drug.

BPs act through the inhibition of bone resorption and are used in combination with antineoplastic chemotherapy for treatment of hypercalcaemia associated with malignancy, lytic bone metastasis and multiple myeloma (3). Several cases describing a correlation between osteonecrosis of the jaws and intravenous administration of BPs have been recently reported in literature $(4,5)$.

Different definitions for ONJ have been proposed and all include exposure of maxillary or mandibular bone, but a breach in the oral mucosa is an absolute requirement. At the present time, it is in fact, not definitively known if the ONJ lesion originates in the bone, or whether it may initiate in the oral mucosa.

The clinical examination of patients needs to be accompanied by a careful evaluation of the imaging features of bone lesions to better understand their extent and features. Digital panoramic radiography, computed tomography (CT) scan, magnetic resonance imaging (MRI) and 99Tcm-MDP three-phase bone scan are diagnostic tools that add value to the clinical findings by revealing different aspects of bone involvement.

Since 2003, numerous reports of bisphosphonate-induced osteonecrosis of the jaws have been reported in literature (6). Several theories have been proposed regarding the etiopathology of ONJ, e.g. that necrosis is related to an over suppression of bone turnover by BPs $(7,8)$ or that BPs decrease angiogenesis (9-11).

The jaw bones are separated from a trauma-intense and microbiologically diverse oral environment by thin mucosa 
and periosteum. Minor trauma may cause local damage to the thin barrier, leading to bone necrosis. Besides, teeth are usually infected by bacteria that cause caries or periodontal disease and are separated from periodontal tissue $\sim 2 \mathrm{~mm}$. This condition allows the access of the infection to underlying bone (12) coupled with chronic invasive dental treatments and the thin mucosa over bone, this anatomical concentration of BPs causes this condition to be manifested exclusively in the jaws. Oral infections are a considerable problem in patients with cancer treated with intensive chemotherapy regimens, including hematopoietic stem-cell transplant procedures. These infections are caused by the complex interaction between the toxicity of cancer chemotherapy for oral mucosal tissue.

Thus, the aim of the present study was to evaluate human gingival epithelium and underlying bone after BPs administration and to analyze the structural damage of the mucosa in ONJ patients correlated to damage of the bone. We examined the effects of zoledronate and aledronate, nitrogen-containing BPs, on human oral mucosa and the underlying mandibular bone.

We demonstrated that BPs induce changes in expression of adhesion to cell-cell/cell-matrix proteins in human oral mucosa. In addition, we demonstrated that a gradual decrease of adhesion proteins correlate with bone damage.

\section{Materials and methods}

Samples of human gingival epithelium and bone tissue were obtained from 12 patients, 6 affected by ONJ and treated with alendronate orally administered (70 $\mathrm{mg}$ for week), and 6 affected by cancer as well as breast or prostatic cancer treated with zoledronate (4 $\mathrm{mg}$ for month) by intravenous infusion over at least $15 \mathrm{~min}$. Both BPs have a light chain attached to the central carbon atom increasing efficacy of drug but also its toxicity.

All patients showed exposing bone areas of jaw. Biopsies were obtained during sequestrectomy intervention removing necrotic bone and relative perilesional gingival mucosa.

In another group of subjects who had undergone oral procedures for other reasons, not treated with BPs, intrasurgical biopsies were obtained and then used as normal control.

The age of the patients ranged between 30 and 81 years and all gave their informed consent. The procedures followed were in accordance with the principles outlined in the Helsinki Declaration of 1975.

The biopsies were treated to study the bone tissue by scanning electron microscopy and the gingival mucosa by immunohistochemistry method.

Scanning electron microscopy. The biopsy specimens utilized for the scanning electron microscopy were fixed for $24 \mathrm{~h}$ in $2.5 \%$ glutaraldehyde in $0.1 \mathrm{M}$ phosphate buffer at $\mathrm{pH} 7.4$ at room temperature. The specimens were dehydrated through a gradual increase in concentrations of ethanol and amile acetate; they were then dried at critical-point in a Balzers critical point drier using liquid $\mathrm{CO}_{2}$. The fractured surfaces of bone were then mounted on stub and platinum coated with a sputtering system 'Plasma Sciences CrC-100 Turbo
Pumped' and observed by Phenom G2 pro scanning electron microscope.

Immunohistochemistry. Biopsies of gingival mucosa were fixed in $3 \%$ paraformaldehyde in $0.2 \mathrm{M}$ phosphate buffer, $\mathrm{pH} 7.4$, for $2 \mathrm{~h}$ at room temperature. They were then washed extensively with $0.2 \mathrm{M}$ phosphate buffer, $\mathrm{pH}$ 7.4, and then with phosphate-buffered saline (PBS), containing 12 and $18 \%$ sucrose. The samples were snap-frozen in liquid nitrogen and $20 \mu \mathrm{m}$ sections were prepared in a cryostat for their use in a protocol for immunofluorescence. The sections were placed on glass slides that were coated with $0.5 \%$ gelatin and $0.005 \%$ chromium potassium sulphate. To block non-specific binding sites and to permeabilize the membranes, the sections were preincubated with $1 \%$ bovine serum albumin (BSA), $0.3 \%$ Triton X-100 in PBS at room temperature for 15 min. Finally, the sections were incubated with primary antibodies against sarcoglycans and integrins in order to value adhesion zones cell-cell and cell-extracellular matrix of gingival mucosa.

The following antibodies for double fluorescence were used: anti- $\alpha$-sarcoglycan diluted 1:100, anti- $\beta$-sarcoglycan diluted $1: 200$, anti- $\gamma$-sarcoglycan diluted 1:100, anti- $\delta$-sarcoglycan diluted 1:50, anti- - -sarcoglycan diluted 1:100 (all from Novocastra Laboratories, Newcastle upon Tyne, UK); anti- $\beta 1$ integrin diluted 1:100, and anti- $\alpha 6$-integrin diluted 1:100 (both from Sigma Chemicals, St. Louis, MO, USA); anti- $\alpha 2$-integrin diluted 1:100 (Jackson ImmunoResearch Laboratories, West Grove, PA, USA). In all reactions, Texas Red conjugated IgG anti-goat (red channel), and FITCconjugated IgG antimouse (green channel), all from Jackson ImmunoResearch Laboratories, were used respectively.

Slides were finally washed in PBS and sealed with mounting medium. The sections were then analyzed and images acquired using a Zeiss LSM 5 DUO confocal laser scanning microscope by META module. All images were digitalized at a resolution of 8 bits into an array of $2048 \times 2048$ pixels. Optical sections of fluorescent specimens were obtained using a HeNe laser (wavelength, $543 \mathrm{~nm}$ ) and an Argon laser (wavelength, $458 \mathrm{~nm}$ ) at a 1-min 2-sec scanning speed with up to 8 averages; $1.50 \mu \mathrm{m}$-thick sections were obtained using a pinhole of 250. Contrast and brightness were established by examining the most brightly labeled pixels and choosing the settings that allowed clear visualization of the structural details while keeping the pixel intensity at its highest $(200)$.

Each image was acquired within $62 \mathrm{sec}$, in order to minimize photodegradation. Digital images were cropped and the figure montage prepared using Adobe Photoshop 7.0.

\section{Results}

Scanning electron microscopy. On healthy subject, the observation of the fracture surface in biopsy of the jaw bone, show the presence of bone lamellae parallel to each other and partially overlapping like roof tiles, alternating to bone lamellae with the same architecture, but with opposite orientation (Fig. 1A). A higher magnification allow to show, in each flap, bands of fibrillar subunits with parallel arrangement to each other and parallel to the major axis of the lamellae (insert in Fig. 1A). On a fracture in orthogonal plane to that previously described, are several furrows transversely oriented to the major axis of 

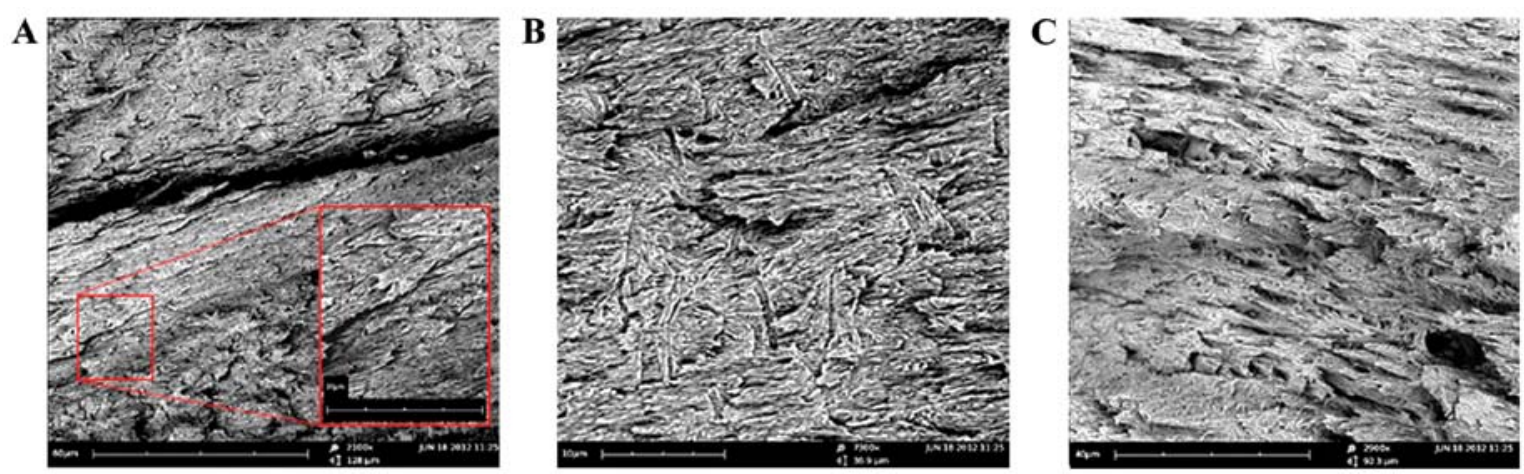

Figure 1. Compound panel showing findings in fracture surfaces of mandibular biopsy in subject untreated with bisphosphonates. (A) Bony lamellae parallel to each other and partially overlapping like roof tiles are visible; higher magnification allow to show that these lamellae alternate to similar bone lamellae with opposite orientation (insert in A). (B) Several furrows transversely orientating to major axis of lamellae and bifurcating to Y are present. (C) Higher magnification allow to better denote the morphology of furrows with fibrillar subunits.
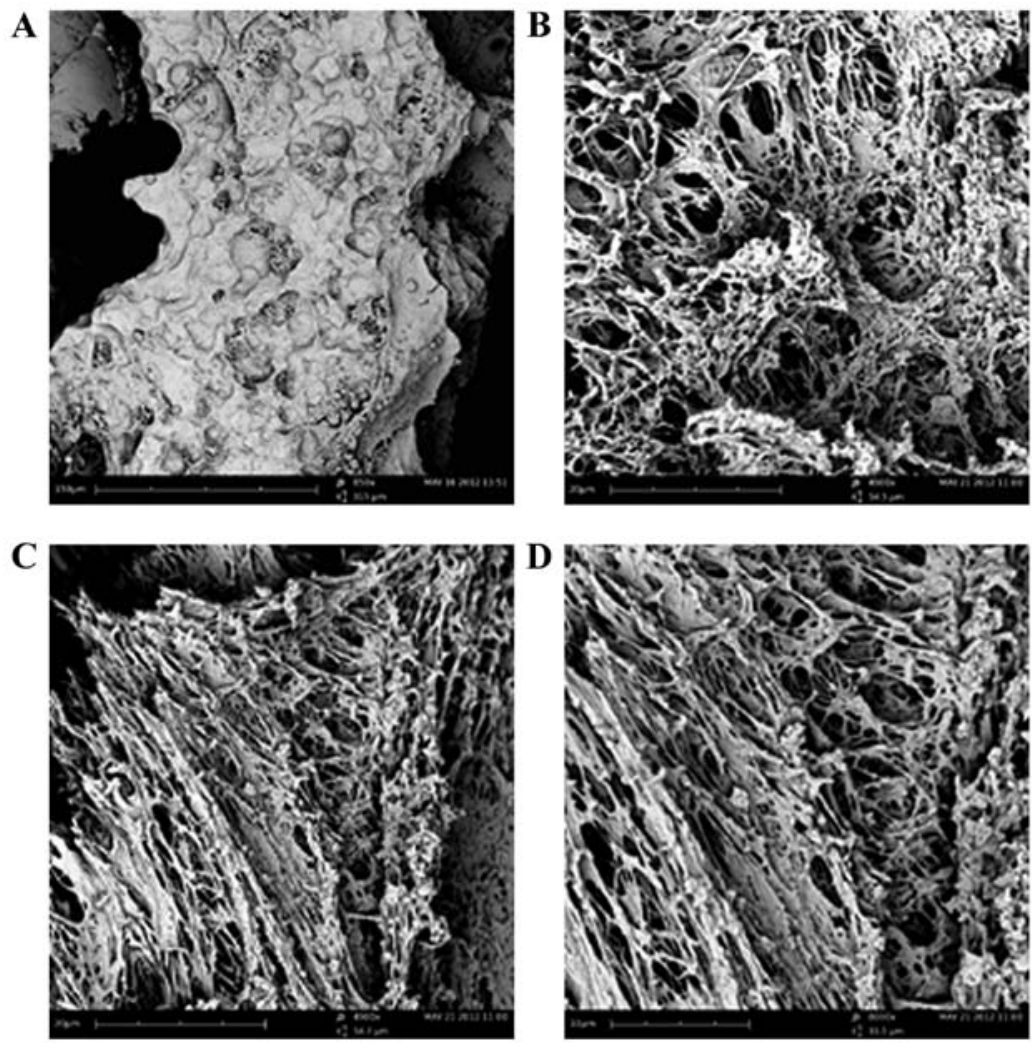

Figure 2. Compound panel showing findings in fracture surfaces of mandibular biopsy in subject treated with bisphosphonates for 24 months. (A) A honeycomb morphology with half-cells irregularly spherical is visible. (B) At higher magnification fibrillar subunits constituting surface of half-cells are irregularly arranged; (C) occasionally, these subunits show a parallel orientation, (D) more visible at further high magnification.

lamellae, which frequently bifurcate to Y (Fig. 1B); at higher magnification, the morphology of the furrows with fibrillar subunits of the lamellae can be clearly seen (Fig. 1C).

In subjects treated with BPs for 24 months, the fracture surfaces of biopsy fragments of mandibular bone frequently show areas with honeycomb morphology represented by halfcells irregularly spherical or elongated and interrupted by presence of half-cells of smaller size (Fig. 2A).

Higher magnification allows to distinguish the fibrillar subunits irregularly arranged or (Fig. 2B) oriented parallel (Fig. 2C), constituting the surface of half-cells. Further high magnification permits individual fibrillar subunits to be observed, sized $\sim 0.001 \mathrm{~mm}$ (Fig. 2D).

In patients treated with BPs for 36 months visible extensive and frequent areas consisting of a honeycomb structure, or areas with half-cells of different sizes and irregular boundaries, occasionally, partially overlapping each other can be seen (Fig. 3A). Sometimes, the bottom of the half-cells appears compact, although it is possible to recognize the presence of fibrillar bands (insert in Fig. 3A).

In other cases the bottom, as well as the outline, appear clearly fibrillar (Fig. 3B); in some areas, it is possible to observe 

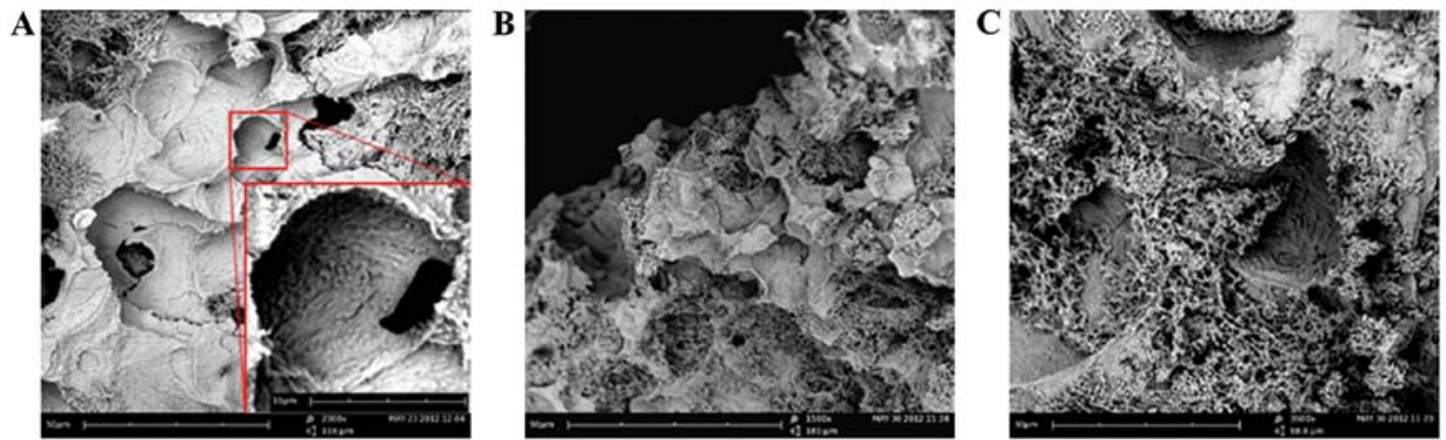

Figure 3. Compound panel showing findings in fracture surfaces of mandibular biopsy in subjects treated with bisphosphonates for 36 months. (A) Areas with honeycomb structure are more extensive and the half-cells show different sizes and more irregular boundaries; occasionally, the bottom of half-cells appears compact, but fibrillar structure is visible (insert in A). (B) Moreover, the half-cells are clearly fibrillar or (C) it is possible to observe a total arrangement of the fibrillar component.
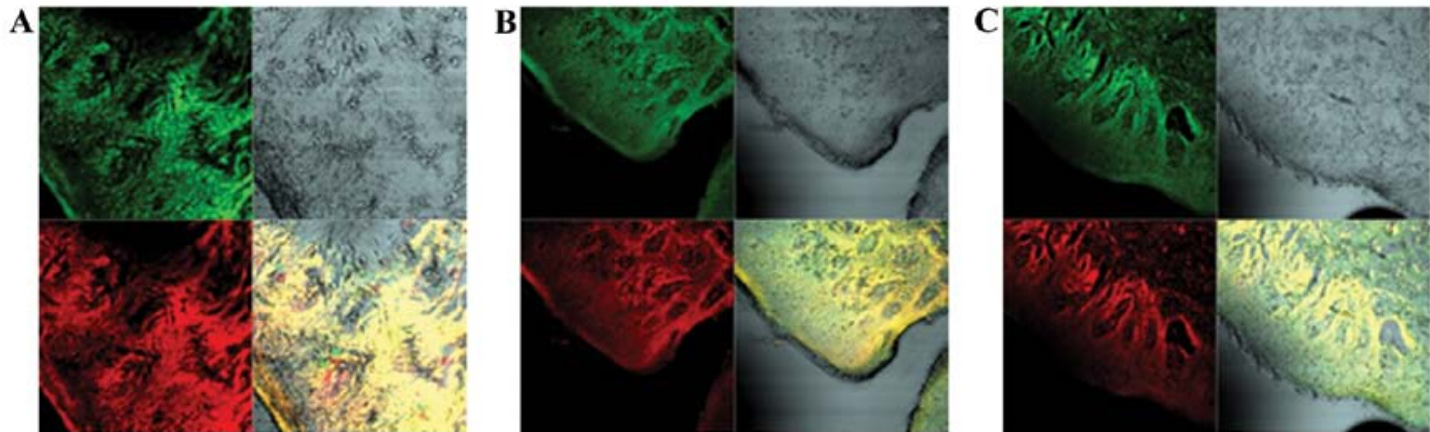

Figure 4. Compound panel showing immunohistochemical findings in gingival mucosa of subjects untreated with bisphosphonates. Double localization reactions between sarcoglycans and integrins show a normal staining pattern in all analyzed regions. By splitting the image it is possible to observe a normal staining patterns for $\alpha$-sarcoglycan (red channel in A) and $\beta 1$-integrin (green channel in A), while the merge, showing a yellow fluorescence, allows to denote a colocalization between two tested proteins. The same behavior of staining patterns is visible for $\varepsilon$-sarcoglycan (red channel in B) and $\alpha 6$-integrin (green channel in B) and for $\gamma$-sarcoglycan (red channel in C) and $\alpha 2$-integrin (green channel in C).
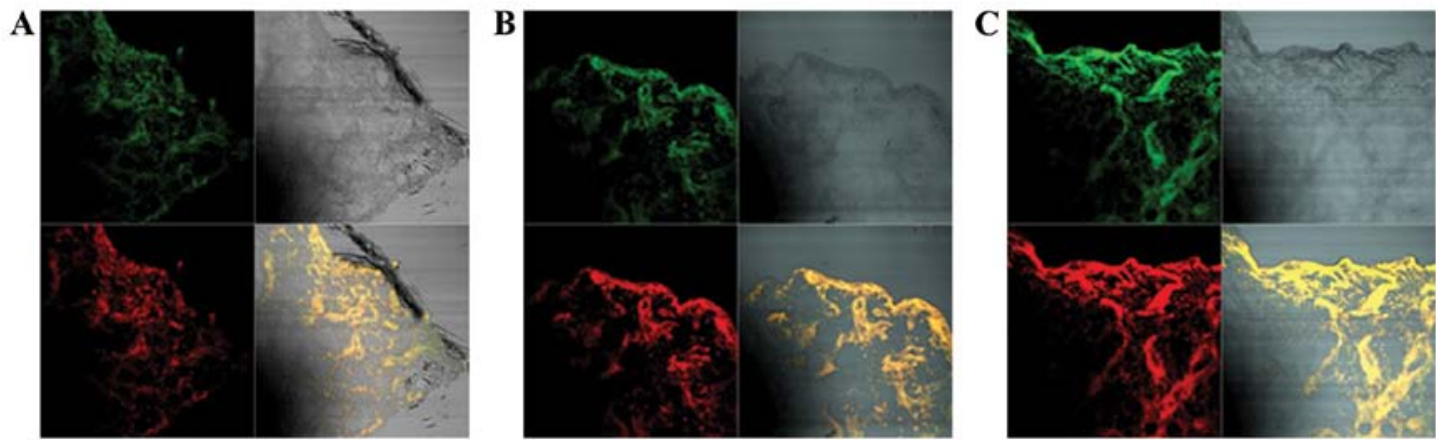

Figure 5. Compound panel showing immunohistochemical findings in gingival mucosa of subjects treated with bisphosphonates for 24 months. Double localization reactions between sarcoglycans and integrins show a decreased staining patterns in respect to normal subjects. By splitting the image, it is possible to observe a decreased staining pattern for $\alpha$-sarcoglycan (red channel in A) and $\beta 1$-integrin (green channel in A), while the merge of two channels, showing a yellow fluorescence, allows to denote a colocalization between two tested proteins. The same behavior of staining patterns is visible for $\varepsilon$-sarcoglycan (red channel in B) and $\alpha 6$-integrin (green channel in B) and for $\gamma$-sarcoglycan (red channel in C) and $\alpha 2$-integrin (green channel in C).

a total derangement of fibrillar component to the bottom of the half-cells (Fig. 3C).

Immunohistochemistry. Double localization reactions between sarcoglycans and integrins, on intraoperative biopsies of oral mucosa of subjects not treated with BPs demonstrate a normal staining pattern in all analyzed regions from basal keratinocytes to superficial layers. By splitting the image it is possible to denote staining patterns for $\alpha$-sarcoglycan and $\beta 1$-integrin (red and green channel in Fig. 4A, respectively), the merging of the channels shows a yellow fluorescence demonstrating a colocalization between two tested proteins. Similar staining patterns are shown for $\varepsilon$-sarcoglycan and a6-integrin (red and green channel in Fig. 4B, respectively) and for $\gamma$-sarcoglycan and $\alpha 2$-integrin (red and green channel in Fig. 4C, respectively). 

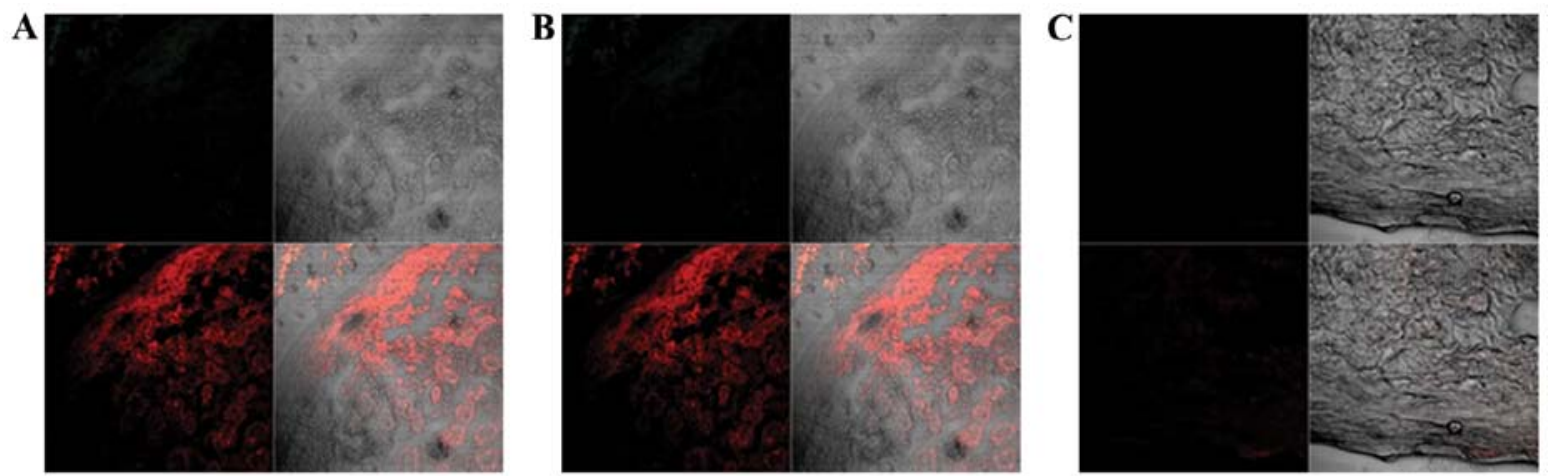

Figure 6. Compound panel showing immunohistochemical findings in gingival mucosa of subjects treated with bisphosphonates for 36 months. Double localization reactions between sarcoglycans and integrins show an absence of staining pattern for tested proteins. By splitting the image, it is possible to denote a markedly decreased staining patterns for $\alpha$-sarcoglycan (red channel in A) and the absence for $\beta 1$-integrin. (B) Analysis of $\varepsilon$-sarcoglycan and $\alpha 6$-integrin and (C) of $\gamma$-sarcoglycan and $\alpha 2$-integrin indicate absence of staining pattern.

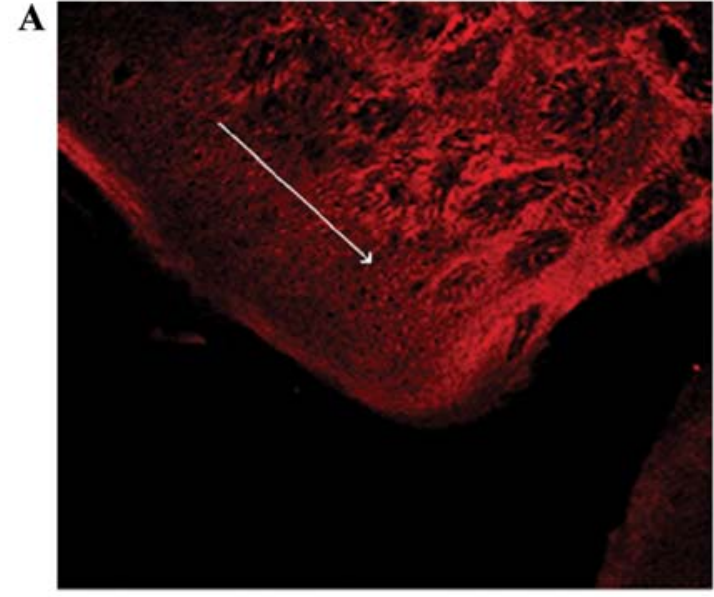

\section{Intensity}

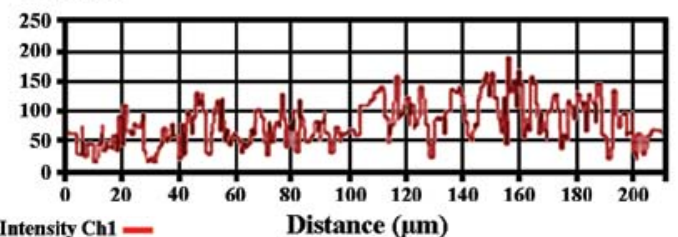

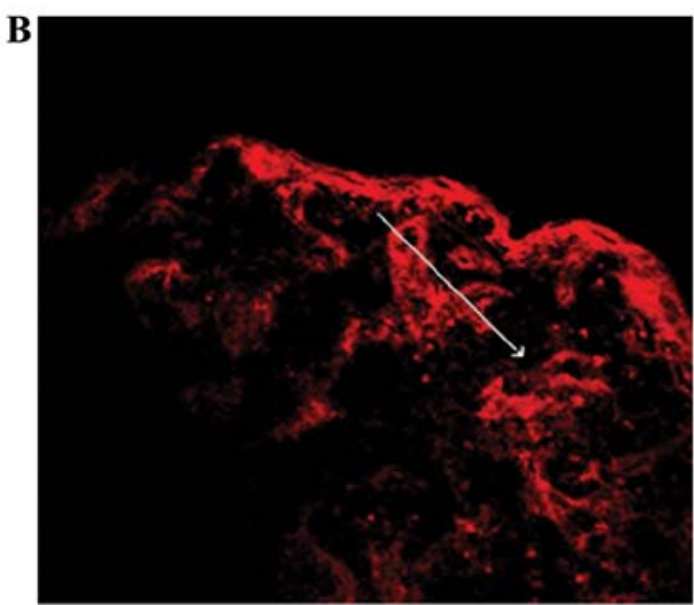

Intensity

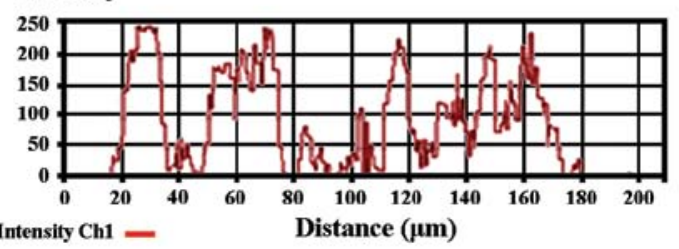

Figure 7. Display profiles of gingival mucosa. (A) In biopsies of subjects untreated with bisphosphonates the analysis shows clear and frequent peaks of fluorescence for $\varepsilon$-sarcoglycan; (B) on biopsy of patients treated with bisphosphonates for 24 months peaks of fluorescence appear less frequent, demonstrating a decreased fluorescence.

Double localization reaction between sarcoglycans and integrin in intraoperative biopsies of the oral mucosa in patients treated with zoledronate for 24 months, compared with healthy subjects, generally show a decreased staining pattern for both proteins. In detail, by splitting the image it is possible to show decreased staining patterns for $\alpha$-sarcoglycan and $\beta 1$-integrin (red and green channel in Fig. 5A, respectively), while the merge of two channels shows a colocalization between two tested proteins. Similarly, the staining patterns for $\varepsilon$-sarcoglycan and $\alpha 6$-integrin (red and green channel in Fig. $5 \mathrm{~B}$, respectively) and for $\gamma$-sarcoglycan and $\alpha 2$-integrin (red and green channel in Fig. 5C, respectively), show decreased fluorescence.

On intraoperative biopsies of the oral mucosa of patients undergoing BPs therapy for 36 months, double localization reaction between sarcoglycans and integrins reveals an almost absent staining pattern for the tested proteins. By splitting of image it is possible to denote the absence of $\beta 1$-integrin (Fig. 6A) and a markedly decreased staining pattern for $\alpha$-sarcoglycan (red channel in Fig. 6A). Staining patterns for $\varepsilon$-sarcoglycan and $\alpha 6$-integrin (red and green channel in Fig. 6B, respectively) and for $\gamma$-sarcoglycan and $\alpha 2$-integrin (red and green channel in Fig. 6C, respectively), show absence of the fluorescence.

To confirm the protein staining patterns, we used the 'display profile' software function of the laser scanning microscope for selected samples. This additional analysis, which reveals the fluorescence intensity profile across an image along a freely selectable line, converted the immunofluorescence signal into a graph. The display profile of the control 


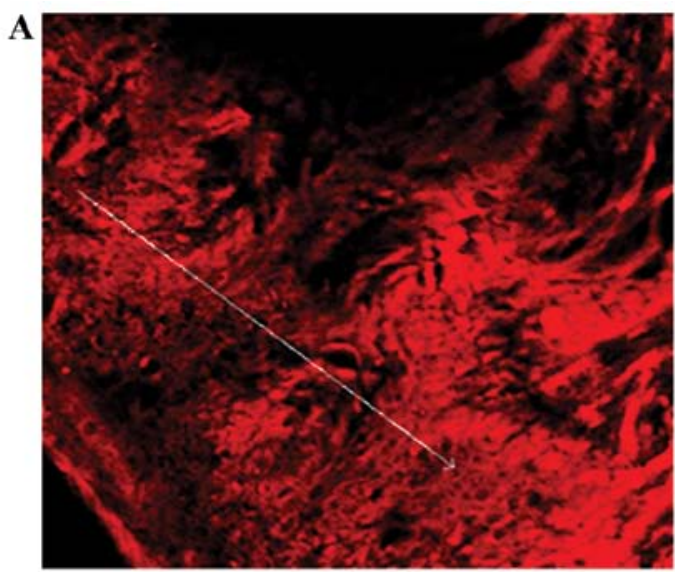

Intensity

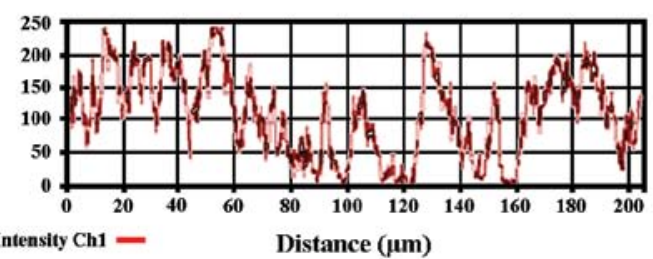

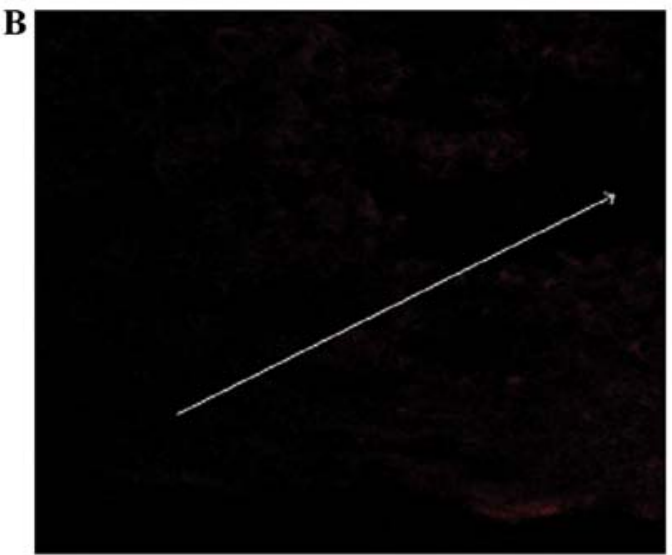

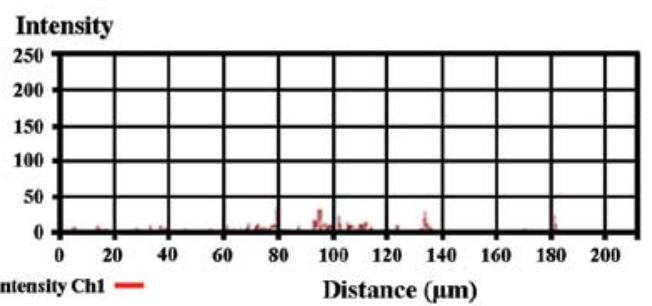

Figure 8. Display profiles of gingival mucosa. (A) In biopsies of subjects untreated with bisphosphonates this analysis shows clear and frequent peaks of fluorescence for $\alpha$-sarcoglycan; (B) on biopsy of patients treated with bisphosphonates for 36 months it is possible to denote an almost absence of peaks of fluorescence.

specimens shows clear and frequent peaks of fluorescence for $\varepsilon$-sarcoglycan (Fig. 7A), while a biopsy of a patient treated with BPs for 24 months, peaks of fluorescence appear less frequently, demonstrating a decreased fluorescence (Fig. 7B). In the same way, the display profile for $\alpha$-sarcoglycan, in a control subject, shows normal and frequent peaks of fluorescence (Fig. 8A), while a biopsy treated with BPs for 36 months shows an almost total absence of peaks (Fig. 8B).

\section{Discussion}

Osteonecrosis of the jaw has been identified as a serious potential complication during prolonged therapy with BPs $(6,13)$. Many studies have suggested that zoledronate and pamidronate may be directly responsible for bisphosphonates related osteonecrosis of the jaw $(14,15)$; nevertheless, the exact mechanism of a predilection for the jaw remains unclear.

Regarding bone healing it was hypothesized that BPs have an effect on bone blood flow in rats and significantly they also reduce circulating vascular endothelial growth factor levels; in other words, the BPs inhibit the angiogenesis process $(16,17)$.

In particular, anti-angiogenic properties have been reported for nitrogen-containing BPs and decreased levels of vascular endothelial growth factor have been observed in humans after administration of zoledronic acid $(9,10,18)$.

This hypothesis has been contrasted in several cases of ONJ, reporting intact vascular channels, even in areas with acute inflammatory infiltrates and bacterial over growth; moreover, no-vital bone fragments without any vascular alteration were also described (19). Recently, the increased level of VEGF in patients with ONJ was demonstrated, using immunohistochemistry methods (11).
An alternative hypothesis is that BPs directly affect viability of osteocytes decreasing their lifespan and increasing the rate of necrotic matrix formation.

In contrast, there is no evidence on the in vivo effects of BPs on osteocytes at high doses, although pamidronate and alendronate have been show to inhibit osteoblastic differentiation in vitro and to suppress the activity of osteoblasts in vivo (20-22). It is possible that during prolonged therapy the BPs may accumulate in the bone toxic levels for osteocytes. Particularly, the mandible and maxillary bone have high rates of bone remodeling and are sites of high rate BP uptake and accumulation.

It is known that the BPs inhibit the mevalonate pathway; blocking the enzyme farnesyl-diphosphate synthase they lead to poor cell function and apoptosis $(23,24)$. In this case, the osteocytes move down by howship lacunae; the lacunae appear empty because the osteoclasts cannot produce new bone material. In our study, at 24 months of treatment with BPs the perilesional bone demonstrated delineated areas with micro-lacunae; after 36 months of treatment the micro-lacunae became wide areas.

Iwata et al (21) have valued connection between organic matrix necrosis toxicity of BPs towards the osteocytes. Moreover, it is not clear whether osteocytic death in the canine model occurs through direct toxic effects of BPs or it occurs through reduced/suppressed remodeling of regions which normally undergo cell death. Which of these hypothesis is correct remains an open question; in either cases, the osteocyte death without replacement inevitably leads to bone matrix necrosis. Our reports of human perilesional bone tissue demonstrate wide areas with absence of matrix and the presence of fibrillar structures exclusively. At 36 months of treatment with BPs we observed many unorganized fibrils or crushed fibrils. 
It was proposed that higher BP levels in alveolar bone would result in high enough $\mathrm{BP}$ concentrations to be directly toxic to oral epithelium, resulting in secondary infection of the underlying bone (25). Our findings demonstrate a strong correlation between gingival epithelium alteration and bone alteration. In agreement, Reid et al (26) suggested that seminal event in the development of ONJ was actually bisphosphonate-induced soft tissue injury. Indeed, it is known that the BPs as pamidronate and aledronate have cytotoxic effects on human intestinal cells (27,28). In particular, Twiss et al (29) demonstrated increased cell permeability after high doses of pamidronate and aledronate exposure in vitro.

Landesberg et al (30) evaluated whether oral keratinocytes undergo apoptosis following exposure to pamidronate by three different methods. But all assays failed to show any significant increase in oral keratinocyte apoptosis secondary to BP exposure. However, it was demonstrated that high concentrations show a significant decreased cellular adhesion in the keratinocytes. This decrease in cell-cell adhesion could create cellular necrosis.

In the present study, we have demonstrated that the subjects treated with BPs, independently by adopted drug, reveal a gradual decrease or absence of transmembrane proteins of integrin system and sarcoglycan complex. This decrease is highly correlated with the treatment duration. It is known that integrins and sarcoglycans are transmembrane proteins that can have a role in cell-cell adhesion and in cell-matrix adhesion (32-34). Therefore, we hypothesize that the BPs have an effect on development of ONJ in two different ways: a direct one on the bone and a indirect one from gingival epithelium. In the direct way on the bone the BPs cause matrix necrosis in maxillar and mandibular bone that have a high bone remodeling from the constant stress of masticatory forces; turnover suppression increases the mean tissue because mandibular bone is remodeled less frequently. In the second way the BPs have effect on the gingival epithelium and they, at the same time, cause absence of cell-cell adhesion and cell-matrix adhesion that lead to cellular apoptosis; this second way facilitates the transit of normal bacterial flora in underlying bone of oral region that causes clinical manifestation as osteomyelitis.

In conclusion, our data show, for the first time, a strong temporal relationship between damage of gingival mucosa and perilesional damage. Regardless of drug chosen and of administration modalities, we demonstrate that both mucosal damage and bone tissue damage increases together with advance of time of treatment.

Primarily, we hypothesize that BPs act on jaw bone through two mechanisms; first, directly on bone tissue, second, indirect on gingival mucosa acting on gingival epithelial permeability. Our results need further analyses on experimental animal models in order to confirm these hypotheses.

\section{References}

1. Merigo E, Manfredi M, Meleti M, et al: Bone necrosis of the jaws associated with bisphosphonate treatment: a report of twentynine cases. Acta Biomed 77: 109-117, 2006.

2. Gutta R and Louis PJ: Methemoglobinemia: an unusual cause of intraoperative hypoxya. Oral Surg Oral Med Oral Pathol Oral Radiol Endod 103: 197-202, 2007.

3. Fleisch H: The use of bisphosphonates in osteoporosis. Br J Clin Pract 48: 323-326, 1994.
4. Wang CJ, Wang JW, Weng LH, Hsu CC, Huang CC and Chen HS: The effect of Alendronate on bone mineral density in the distal part of the femur and the proximal part of the tibia after total knee arthroplasty. J Bone Joint Surg Am 85: 2121-2126, 2003

5. Ficarra G, Beninati F, Rubino I, Vannucchi A, Longo G, Tonelli P and Pini Prato G: Osteonecrosis of the jaws in periodontal patients with a history of bisphosphonate treatment. J Clin Periodontol 32: 1123-1128, 2005

6. Marx RE: Pamidronate (Aredia) and zoledronate (Zometa) induced avascular necrosis of the jaws: a growing epidermic. J Oral Maxillofac Surg 61: 1115-1157, 2003.

7. Ruggiero SL, Mehrotra B, Rosenberg TJ and Engroff SL: Osteonecrosis of the jaws associated with the use of bisphosphonates: a review of 63 cases. J Oral Maxillofac Surg 62: 527-534, 2004.

8. Marx RE, Sawatari Y, Fortin M and Broumand V: Bisphosphonate-induced exposed bone (osteonecrosis/osteoporosis) of the jaws: risk factors, recognition, prevention, and treatment. J Oral Maxillofac Surg 63: 1567-1575, 2005.

9. Wood J, Bonjean K, Ruetz S, et al: Novel antiangiogenic effects of the bisphosphonate compound zoledronic acid. J Pharmacol Exp Ther 302: 1055-1061, 2002.

10. Santini D, Vincenzi B, Dicuonzo G, et al: Zoledronic acid induces significant and long-lasting modifications of circulating angiogenic factors in cancer patients. Clin Cancer Res 9: 2893-2897, 2003.

11. Nastro Siniscalchi E, Cutroneo G, Catalfamo L, et al: Immunohistochemical evaluation of sarcoglycans and integrins in gingival epithelium of multiple myeloma patients with bisphosphonate-induced osteonecrosis of the jaw. Oncol Rep 24: 129-134, 2010.

12. Woo SB, Hellstein JW and Kalmar JR: Narrative (corrected) review: BPs and osteonecrosis of the jaws. Ann Intern Med 144: 753-761, 2006.

13. Mehrotra B and Ruggiero S: Bisphosphonate complications including osteonecrosis of the jaw. Hematology Am Soc Hematol Educ Program 515: 356-360, 2006.

14. Migliorati CA, Casiglia J, Epsten J, Jacobsen PL, Siegel MA and Woo SB: Managing the care of patients with bisphosphonateassociated osteonecrosis: an American Academy of Oral Medicine position paper. J Am Dent Assoc 136: 1658-1668, 2005.

15. Khosla S, Burr D, Cauley J, et al: Bisphosphonate-associated osteonecrosis of the jaw: report of a task force of the American Society for Bone and Mineral Research. J Bone Miner Res 22: 1479-1489, 2007.

16. Cheng A, Mavrokokki A, Carter G, et al: The dental implications of bisphosphonates and bone disease. Aust Dent J 50: S4-S13, 2005.

17. Kapitola J and Zák J: Effect of pamidronate on bone blood flow in oophorectomized rats. Physiol Res 47: 237-240, 1998.

18. Allegra A, Oteri G, Nastro E, et al: Patients with bisphosphonateassociated osteonecrosis of the jaw have reduced circulating endothelial cells. Hematol Oncol 25: 164-169, 2007.

19. Hellstein JW and Marek CL: Bisphosphonate osteochemonecrosis (bis-phossy jaw): is this phossy jaw of the 21st century? J Oral Maxillofac Surg 63: 682-689, 2005.

20. Idris AI: Role of cannabinoid receptors in bone disorders: alternatives for treatment. Drug News Perspect 21: 533-540, 2008.

21. Iwata K, Li J, Follet H, Phipps RJ and Burr DB: Bisphosphonates suppress periosteal osteoblast activity independently of resorption in rat femur and tibia. Bone 39: 1053-1058, 2006.

22. Tobias JH, Chow JW and Chambers TJ: 3-Amino-1hydroxypropylidine-1-bisphosphonate (AHPrBP) suppresses not only the induction of new, but also the persistence of existing bone-forming surfaces in rat cancellous bone. Bone 14: 619-623, 1993.

23. Shipman CM, Rogers MJ, Apperley JF, Russell RG and Croucher PI: Bisphosphonates induce apoptosis in human myeloma cell lines: a novel anti-tumour activity. Br J Haematol 98: 665-672, 1997.

24. van Beek ER, Cohen LH, Leroy IM, Ebetino FH, Löwik CW and Papapoulos SE: Differentiating the mechanisms of antiresorptive action of nitrogen containing bisphosphonates. Bone 33: 805-811, 2003.

25. Bauss F and Bergström B: Preclinical and clinical efficacy of the bisphosphonate ibandronate in cancer treatment. Curr Clin Pharmacol 3: 1-10, 2008

26. Reid IR, Bolland MJ and Grey AB: Is bisphosphonate-associated osteonecrosis of the jaw caused by soft tissue toxicity? Bone 41: 318-320, 2007. 
27. Twiss IM, de Water R, den Hartigh J, et al: Cytotoxic effects of pamidronate on monolayers of human intestinal epithelial (Caco-2) cells and its epithelial transport. J Pharm Sci 83: 699-703, 1994.

28. Suri S, Monkkonen J, Taskinen M, Pesonen J, Blank MA, Phipps RJ and Rogers MJ: Nitrogen-containing bisphosphonates induce apoptosis of Caco- 2 cells in vitro by inhibiting the mevalonate pathway: a model of bisphosphonate-induced gastrointestinal toxicity. Bone 29: 336-343, 2001.

29. Twiss IM, Pas O, Rampkoopmanscap W, den Harigh J and Vermeij P: The effects of nitrogen-containing bisphosphonates on human epithelial (Caco-2) cells, an in vitro model for intestinal epithelium. J Bone Miner Res 14: 784-791, 1999.

30. Landesberg R, Cozin M, Cremers S, Woo V, Koustent S and Sinba S: Inhibition of oral mucosal cell wound healing by bisphosphonates. J Oral Maxillofac Surg 66: 839-847, 2008.
31. Anastasi G, Amato A, Tarone G, et al: Distribution and localization of vinculin-talin integrin system and dystrophin-glycoprotein complex in human skeletal muscle: immunohistochemical study using confocal laser scanning microscopy. Cells Tissues Organs 175: 151-164, 2003.

32. Anastasi G, Cutroneo G, Rizzo G, et al: Sarcoglycan and integrin behavior in normal human skeletal muscle: a CLSM (confocal laser scanning microscope) study. Eur J Histochem 48: 245-252, 2004.

33. Anastasi G, Cutroneo G, Santoro G, et al: Integrins, muscle agrin and sarcoglycans during muscular inactivity conditions: an immunohistochemical study. Eur J Histochem 50: 327-336, 2006.

34. Anastasi G, Cutroneo G, Santoro G, et al: Costameric proteins in human skeletal muscle during muscular inactivity. J Anat 213: 284-295, 2008. 